\title{
Review Article \\ Role of the Ubiquitin-Proteasome Systems in the Biology and Virulence of Protozoan Parasites
}

\author{
Christian Muñoz, Juan San Francisco, Bessy Gutiérrez, and Jorge González \\ Molecular Parasitology Unit, Medical Technology Department, Faculty of Health Sciences, University of Antofagasta, \\ P.O. Box 170, Antofagasta, Chile
}

Correspondence should be addressed to Jorge González; jorge.gonzalez@uantof.cl

Received 1 August 2014; Accepted 6 October 2014

Academic Editor: Luis I. Terrazas

Copyright (C) 2015 Christian Muñoz et al. This is an open access article distributed under the Creative Commons Attribution License, which permits unrestricted use, distribution, and reproduction in any medium, provided the original work is properly cited.

In eukaryotic cells, proteasomes perform crucial roles in many cellular pathways by degrading proteins to enforce quality control and regulate many cellular processes such as cell cycle progression, signal transduction, cell death, immune responses, metabolism, protein-quality control, and development. The catalytic heart of these complexes, the $20 \mathrm{~S}$ proteasome, is highly conserved in bacteria, yeast, and humans. However, until a few years ago, the role of proteasomes in parasite biology was completely unknown. Here, we summarize findings about the role of proteasomes in protozoan parasites biology and virulence. Several reports have confirmed the role of proteasomes in parasite biological processes such as cell differentiation, cell cycle, proliferation, and encystation. Proliferation and cell differentiation are key steps in host colonization. Considering the importance of proteasomes in both processes in many different parasites such as Trypanosoma, Leishmania, Toxoplasma, and Entamoeba, parasite proteasomes might serve as virulence factors. Several pieces of evidence strongly suggest that the ubiquitin-proteasome pathway is also a viable parasitic therapeutic target. Research in recent years has shown that the proteasome is a valid drug target for sleeping sickness and malaria. Then, proteasomes are a key organelle in parasite biology and virulence and appear to be an attractive new chemotherapeutic target.

\section{Introduction}

In a paper published in 1978, Ciehanover et al. [1] reported the presence of a heat-stable polypeptide component of an ATPdependent proteolytic system isolated from reticulocytes. A second paper from the same researchers reported that the ATP-dependent conjugation of reticulocyte proteins to the polypeptide was required for protein degradation [2]. Based on these findings, Hershko et al. [3] proposed in 1980 that the ligation of ubiquitin to proteins targets them for degradation by a protease that specifically acts on proteins with several ubiquitin molecules attached $[3,4]$. A "protease," the $26 \mathrm{~S}$ proteasome, was discovered by Hough and colleagues, who reported the identification and characterization of an ATPdependent protease from rabbit reticulocyte lysates [5]. The real impact of this discovery would be dimensioned in the coming decades. In fact, Hershko's work on the ubiquitin enzymes was not only relevant but contributed to opening a new research field that was obscure and unexplored at that time. The 2004 Chemistry Nobel Prize award, conferred to Hershko, Ciechanover, and Rose "for the discovery of ubiquitin-mediated protein degradation," was not only a recognition of these researchers but a recognition of the importance of the ubiquitin-proteasome pathway to the life of the cell and to health, disease, infection, and immunity [6]. Many researchers have contributed to our current knowledge of this biological pathway. Many relevant reviews have been already published. In this context, the main goal of this review is to attract attention to a new role of proteasomes: the biology and virulence of protozoan parasites. General aspects of the ubiquitin-proteasome pathways and inhibitors will be only summarized.

\section{The Ubiquitin-Proteasome System}

The bulk of the turnover of intracellular proteins in eukaryotic cells is carried out by two self-contained proteolytic 
systems, the lysosomes and proteasomes. Most proteins are degraded by the ubiquitin-proteasome system (UPS) [6].

Proteasomes are large complexes that perform crucial roles in many cellular pathways by degrading proteins in the cytosol and nucleus of eukaryotic cells to enforce quality control and regulate many basic cellular processes. Among these processes are progression through the cell cycle, signal transduction, cell death, immune responses, metabolism, protein-quality control, and development, in which proteasomes degrade short-lived regulatory or structurally aberrant proteins [6, 7]. The catalytic heart of these complexes, the $20 \mathrm{~S}$ proteasome, is highly conserved in bacteria, yeast, and humans [8], with simpler versions also found in some Archaea and prokaryotes.

The $20 \mathrm{~S}$ proteasome is a barrel-shaped assembly of 28 protein subunits. It forms a packed particle, a result of axial stacking of two outer $\alpha$ rings and two inner $\beta$ rings made up of seven structurally related $\alpha$ and $\beta$ subunits; the rings form an $\alpha_{1-7} \beta_{1-7} \beta_{1-7} \alpha_{1-7}$ structure. The three subunits of each inner ring contain catalytically active threonine residues at their $\mathrm{N}$ termini and show $\mathrm{N}$-terminal nucleophile hydrolase activity, indicating that the proteasome is a threonine protease [8]. The $\beta 1, \beta 2$, and $\beta 5$ are associated with caspase-like, trypsinlike, and chymotrypsin-like activities, respectively, which confer the ability to cleave peptide bonds at the C-terminal side of acidic, basic, and hydrophobic amino acid residues, respectively. Those bonds that follow glycine and proline are less easily cleaved [9]. As revealed by structural studies performed by Huber and colleagues [10, 11], the potentially catastrophic elimination of inappropriate substrates is prevented by sequestration of active sites within the hollow structure of the $20 \mathrm{~S}$ proteasome. Substrates access the central catalytic chamber through axial ports in the end rings of $\alpha$ subunits [12], although in the absence of activators, these channels are closed and proteasome activity is repressed. The 20S proteasome processively degrades client proteins, generating oligopeptides ranking in length from 3 to 15 amino acids. The resulting peptide products are subsequently hydrolyzed to amino acids by oligopeptidases and/or aminocarboxy peptidases [9].

Proteasomes are activated by protein complexes that bind to the end rings of $\alpha$ subunits. The best-known activator is PA700 [proteasome activator MW 700, also known as 19S or regulatory complex $(\mathrm{RC})]$, which has been highly conserved from yeast to humans and binds to the $20 \mathrm{~S}$ proteasome to form the 26S proteasome. PA700 is the only proteasome activator, that is, known to stimulate degradation of protein substrates. Thus, PA700 is thought to mediate most of the biological effects of the proteasome by facilitating substrate degradation $[13,14]$. In contrast to PA700, two other evolutionarily conserved protein complexes that have been shown to bind specifically to and activate $20 \mathrm{~S}$ proteasomes against model peptide substrates, PA28 (also known as 11S or REG) $[7,15]$ and PA200 [7, 16], do not recognize ubiquitinated proteins or use ATP. Proteasome activator PA200 enhances proteasome-mediated cleavage after acidic residues in vitro; however, in response to ionizing radiation, PA200 forms hybrid proteasomes with 195 caps and 20S core proteasomes that accumulate on chromatin, leading to an increase in proteolytic activity. A unique role for PA200 in genomic stability, that is, likely mediated through its ability to enhance post-glutamyl cleavage by proteasomes, has been reported [17]. Blm10/PA200 (Saccharomyces cerevisiae/human) does not utilize ATP and is generally believed to stimulate the hydrolysis of peptides but not proteins. Blm10/PA200 has been proposed to function in a surprisingly broad variety of processes [18], including 20S proteasome assembly [19], DNA repair [20], genomic stability [17], proteasome inhibition [21], spermatogenesis [22], and mitochondrial checkpoint regulation [23]. However, endogenous inhibitors like Hsp 90, P131, PR 39, and Tat have also been described. The biological role of $26 \mathrm{~S}$ proteasomes and its activators and inhibitors have been reviewed extensively elsewhere $[5,7,24$, 25]. New regulatory mechanisms have emerged. The archaeal PAN ATPase complex is homologous to the eukaryotic 19S ATPases and contains a conserved C-terminal hydrophobictyrosine-X motif (HbYX), that is, essential for PAN to associate with the $20 \mathrm{~S}$ proteasomes and open its gated channel for substrate entry [26]. Gate opening can be induced by C-terminal peptides from the $19 \mathrm{~S}$ ATPase subunits, Rpt2, and Rpt5, but not by C-terminal peptides from PA28/26, which lack the HbYX motif and cause gate opening by distinct mechanisms. C-terminal residues in the 19S ATPases were also shown to be critical to the gating and stability of 26S proteasomes. Thus, the $\mathrm{C}$ termini of the proteasomal ATPases function like a "key in a lock" to induce gate opening and allow substrate entry [26]. Recently, it has been shown that binding of polyUb substrates to the $19 \mathrm{~S}$ regulator stabilizes gate opening of the $20 \mathrm{~S}$ proteasome and induces conformational changes in the $20 \mathrm{~S}$ proteasome that facilitate channeling of substrates and their access to active sites. In consequence, polyUb substrates allosterically stimulate their own degradation, enhancing the peptidase activities of the $20 \mathrm{~S}$ proteasome about two-fold in a process requiring ATP hydrolysis [27]. In addition, a recently published body of evidence suggests that many proteasome functions, such as substrate recognition, deubiquitylation, unfolding, and degradation, appear to be controlled allosterically [28, 29].

In this pathway, proteins are targeted for degradation by covalent ligation with ubiquitin. Ubiquitination tags the target protein with ubiquitin-like proteins (UBLs), such as ubiquitin, small ubiquitin-like modifier (SUMO), and NEDD8. Ubiquitination is a posttranslational modification of proteins in which the modifier is a polypeptide conjugated to the target proteins by an isopeptide bond between proteasome substrates: the $\mathrm{C}$ terminus of ubiquitin and one or more lysine side chains in the target proteins [30]. Protein modification by ubiquitin occurs in three successive steps that are mediated by three enzymes: the activating enzyme E1, the conjugating enzyme E2, and the ubiquitin ligase E3. This modification is reversible, and ubiquitinated proteins can be proteolytically deubiquitinated by specific deubiquitinating enzymes $[30,31]$. Ubiquitin molecules can form polyubiquitin chains that are conjugated to target proteins, which are usually recognized and degraded by the proteasome [30, 32]; however, current knowledge of UPS strongly suggests that protein ubiquitination appears to be necessary but not essential. A recent paper reports 
that proteasomes can degrade a significant proportion of cellular proteins independent of ubiquitination. Then, $26 \mathrm{~S}$ proteasomes specifically recognize and cleave similar sites, independent of ubiquitination, suggesting that disordered regions likely constitute the universal structural signal for proteasome-substrate proteolysis by proteasomes. In the same way, the inactivation of ubiquitin-activating enzyme E1 does not prevent intrinsic proteasome substrates degradation [33].

The picture is completed by the deubiquitinating enzymes (DUBs) [30, 34]. They generate free Ub moieties from their initial translation products, recycle ubiquitin during breakdown of the poly-ubiquitin-protein conjugates, and/or reverse the effects of ubiquitination. All DUBs tested have remarkable specificity for ubiquitin. DUBs have been implicated in a variety of processes in animals and yeast, suggesting that individual DUBs are target-specific [34]. An intriguing possibility is that some DUBs can also regulate a protein's half-life by reversing ubiquitination. A large number of genes encode deubiquitinating enzymes, suggesting that many have highly specific and regulated functions. Interestingly, many of these enzymes are localized to subcellular structures or to molecular complexes. These localizations play important roles in determining functional specificity and can have major influences on their catalytic activities [34]. Indeed, recent findings strongly suggest that ubiquitination is regulated by both specific pathways of ubiquitination and deubiquitination. In summary, the protein substrates are first conjugated to multiple molecules of ubiquitin and then ubiquitin substrates are rapidly hydrolyzed by the $26 \mathrm{~S}$ proteasome, an ATP-dependent complex comprising the core $20 \mathrm{~S}$ proteasome enclosed by two proteasome activator (19S) regulatory complexes. Deubiquitination enzymes recycle the ubiquitin molecules and the pathway is modulated by protein activators and inhibitors. An overview of the ubiquitinproteasome system is shown in Figure 1.

In summary, the ubiquitin-proteasome pathway is not only a degradation machine focused to destroy old or damaged proteins. This pathway is a major control point for regulating, among other things, short-lived proteins functioning as regulatory factors in a large array of cellular processes like cell-cycle progression [35], cell growth, stagespecific gene transcription [36], inflammatory response [37], and antigen processing [32]. Eukaryotic 20S proteasomes have several peptidase activities, as well as endoribonuclease, protein-chaperone, and DNA-helicase activities [38].

Until a few years ago, the role of proteasomes in parasite biology was completely unknown.

\section{The Role of Proteasomes in Parasite Biology and Virulence}

Parasitic protozoan are unicellular but complex cells that undergo multiple differentiation events to accommodate the various hosts and physical environments that they encounter in their life cycles.

Some proteases are involved in the differentiation of the infectious stages of a small number of protozoan parasites into their respective disease-causing stages [39-41].

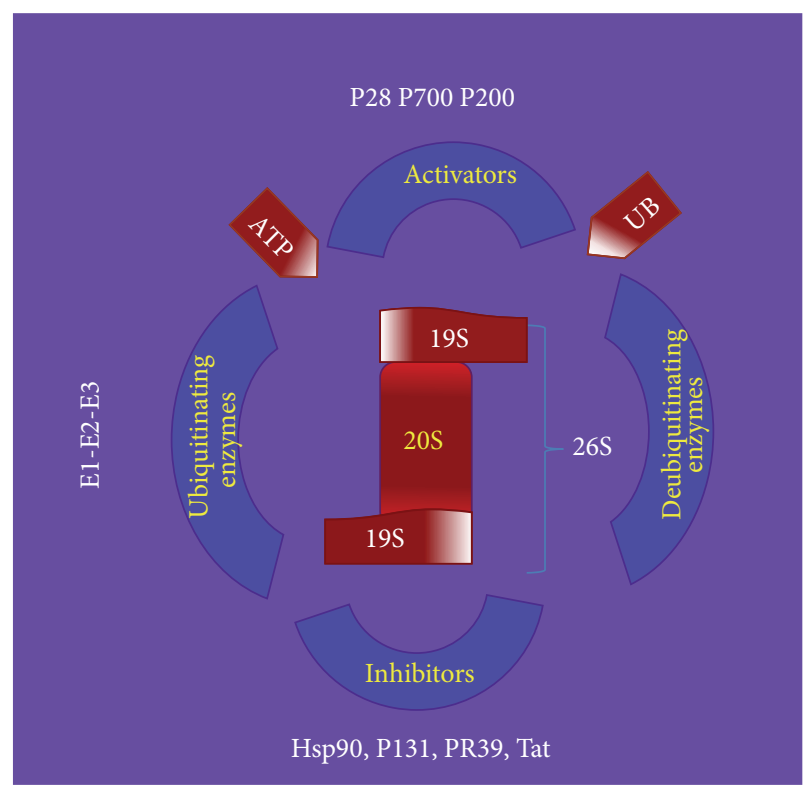

Figure 1: An overview of the main component of ubiquitin proteasome system.

The central role played by the proteolytic activities of the proteasome/ubiquitin system in regulating cell homeostasis has been demonstrated in a large number of fungi and higher eukaryotes and, more recently, in protozoan parasites [42].

A prominent feature of the life cycle of pathogenic parasites is the profound morphological changes they undergo during development in the vertebrate and invertebrate hosts. These developmental changes, during which shape, size, and cytoskeletal structures must adapt to the new stage, involve extensive and carefully controlled proteolysis. The intriguing question of what proteolytic system is involved in protein degradation in parasites led us to investigate the role of proteasomes in differentiation of protozoan parasites.

The protozoan parasites' $20 \mathrm{~S}$ proteasomes are similar in morphology and size to the $20 \mathrm{~S}$ proteasome isolated from archaebacteria, yeast, and mammals. Similarly, the composition of the protozoan proteasomes subunits is very similar to that of the eukaryotic proteasomes, with multiple $\alpha$ and $\beta$ subunits instead of the single type of $\alpha$ and $\beta$ subunit described in Archaebacterias proteasomes. Studies in different laboratories and with different models have found that inhibition of proteasomal function inhibits specific stages of morphological differentiation in Trypanosoma, Plasmodium, and Entamoeba and replication of Plasmodium, Toxoplasma, Leishmania, and Trypanosoma; however, invasion of the host cell is not inhibited in Trypanosoma, Plasmodium, Leishmania, or Toxoplasma. Differences between the proteasomes of mammals and parasites have been observed (Trypanosoma), as have differences in immunoreactive structures (Trypanosoma and Toxoplasma) and enzymatic activities (Trypanosoma and Entamoeba). These differences suggest that protozoan parasite proteasomes could be considered as a chemotherapeutic target, even though this organelle is also present in all host eukaryotic cells. In mammals cells, 
the ubiquitin proteasome system is essential for all eukaryotic cells; any alteration to its components thus has potential pathological consequences [43]. Chemotherapy targeting parasite proteasomes could result in successful therapies.

The following are the main findings reported in the literature with respect to the role of protozoan parasite proteasomes.

3.1. Cryptosporidium. A DNA sequence composed of 1281 nucleotides (nt) consisting of a single open reading frame (ORF) encoding a putative $20 \mathrm{~S}$ proteasome $\beta 1$-type subunit was isolated from Cryptosporidium parvum. Southern-blot analysis suggested that the sequenced DNA exists in the C. parvum genome as a single copy. The predicted protein consists of 210 amino acids (aa), including characteristic amino acids common to all proteasomal subunits, and is more similar to the betal-type subunit of yeast than to other types of beta subunits [44]. No studies have examined its biological role.

3.2. Giardia. The parasite has a single gene that encodes monoubiquitin; however, two-dimensional electrophoresis assays have shown that the Giardia 20S proteasome seems to be as complex as that of other eukaryotes [45]. A study of the seven genes that encode the $\alpha$ subunits of the $G$. duodenalis proteasome indicated that the $\alpha$-proteasome gene family evolved quickly from a single gene in the Archaea to seven or more genes in Eukarya [46]. The G. duodenalis $20 \mathrm{~S}$ proteasome appears to be similar to that described in eukaryotic cells, containing a divergent set of $\alpha$ subunits [47]. Proteomics approaches performed to discover novel proteins associated with the stage-specific, Golgi-like encystationspecific vesicles (ESV) identified cytoplasmic and luminal factors of the endoplasmic reticulum quality-control system, that is, several structural $(\alpha)$ and catalytic $(\beta)$ proteasome subunits. In contrast, cytoplasmic proteasome complexes undergo a developmentally regulated relocalization to ESVs during encystation. In mammalian cells and in yeast, proteasome complexes localize at ER membranes in addition to the cytoplasm and the nucleoplasm. Confocal microscopy analysis demonstrated that the giardial 20S core complex and 19S cap structure were associated with ESV membranes during early encystation until at least $7 \mathrm{~h}$ after induction. As noted previously, the expression of proteasome subunits is not upregulated in encysting cells [47]. The confocal microscopy data indicated a relocalization from more peripheral sites in the cytoplasm to the vicinity of ESVs, indicating a high rate of retrotranslocation of organelle proteins destined for degradation. In light of these results, the authors proposed that proteasome recruitment during encystation is a consequence of quality control and cargo maturation processes in the ER and early ESVs (i.e., protein folding, heterooligomerization, and trimming) producing large amounts of material destined for degradation [48].

3.3. Entamoeba. The $20 \mathrm{~S}$ activity in proteasomes was described based on the SDS-electrophoretic pattern and immunoblot analysis of a soluble Entamoeba histolytica extract fractionated by density-gradient centrifugation [49]. A study of the E. histolytica proteome confirmed the presence of ubiquitin-proteasome components [50]. On the other hand, the genes encoding the $\alpha$ proteasome subunits show a higher identity with mammalian proteasomes $(60.1 \%$ homology with rat proteasomes and $60.5 \%$ with human proteasomes) than with proteasomes from Thermoplasma acidophilum and Saccharomyces cerevisiae $(39.5 \%$ and $53.8 \%$, resp.). In E. histolytica trophozoites, nuclear localization of the $20 \mathrm{~S}$ complex was not evident even by highresolution confocal microscopy [51]. Instead, fluorescent reactivity against the proteasome subunits EhotS and EhS2 was observed exclusively in the cytosol, exhibiting a homogeneous distribution with no apparent exclusion of compartments that resemble the ER and Golgi apparatus, as observed in other cell types $[30,51]$.

Recently, multiple E. histolytica ubiquitination components, including ubiquitin and its activating (E1), conjugating (E2), and ligating (E3) enzymes, have been cloned and characterized. EhUbiquitin is activated by and forms a thioester bond with EhUbal (E1) in vitro in an ATP- and magnesiumdependent fashion. According to the authors, EhUbal exhibits a greater maximal initial velocity of pyrophosphateATP exchange than its human homolog, suggesting that different kinetics of ubiquitin activation might exist in $E$. histolytica [52].

In a reptilian amoeba, Entamoeba invadens, encystation is inhibited by lactacystin, a specific and irreversible inhibitor of proteasomes [53]; however, lactacystin seems to have no effect on E. invadens excystation [54].

3.4. Leishmania. These parasites are protozoan parasites with an intracellular stage called the amastigote that replicates in mammalian macrophages and an extracellular stage called the promastigote that replicates in the intestine of hematophagous insect belonging to genus Lutzomyia.

Purified proteasomes from L. mexicana were studied using polyacrylamide-gel electrophoresis (SDS-PAGE), revealing 10 different bands with masses ranging between 22 and $32 \mathrm{kDa}$, suggesting a complexity similar to that of eukaryotic proteasomes [55]. Lactacystin affected L. mexicana replication only when used at concentrations higher than $5 \mu \mathrm{m}$, while MG132 blocked the same process at lower concentrations. These discrepancies might be due to the lower capacity of $L$. mexicana to incorporate these inhibitors. According to Christensen et al. [56], a new antigen that resembles an $\alpha$ subunit of the human 20S proteasome was identified in Leishmania. This antigen (LePa) is immunogenic in humans. Moreover, a DNA vaccine based on the LePa antigen induced an initial reduction in the size of lesions when mice were challenged with Leishmania major. The strong immunogenicity of the Leishmania proteasome was confirmed by Couvreur et al. [57], who reported that Antigen 24, an immunogenic complex isolated from Leishmania infantum used as reference antigen in the immunodiagnostic of human visceral leishmaniasis, was recognized by the serum of rabbits immunized with purified L. mexicana proteasomes. On the other hand, the Leishmania chagasi proteasome was 
partially purified and showed sensitivity to lactacystin and clasto-lactacystin beta-lactone, which blocked the in vitro growth of the promastigote stage. Although pretreatment of the promastigotes with lactacystin did not inhibit cell invasion, proteasomal function seems to be essential for replication and intracellular survival of amastigotes in the host cell [58].

In synchronized Leishmania cultures, Dubessay et al. [59] reported the cell-cycle-dependent regulation of protein levels. A kinesin called LmjKIN13-1 is highly abundant in the $\mathrm{G} 2+\mathrm{M}$ phase and present at very low levels after mitosis. This protein is degraded through ubiquitinproteasome pathways, demonstrating that it has C-terminal redundant degradation signals. This observation suggests that in Leishmania, in which postranduccional regulation is rare or absent, the proteasome appears to be involved in the regulation of protein levels [59]. On the other hand, in Leishmania donovani, degradation of pteridine reductase 1 (PTR1) has been reported. In Leishmania, PTR1 is an essential enzyme in pterin and folate metabolism. Westernblot studies using L. donovani promastigotes transfected with PTR1-GFP showed that PTR1 was degraded in the stationary phase of growth, when parasites start metacyclogenesis. Similarly, a probable destruction box composed of nine amino acids (Q63ADLSNVAK71) and a lysine K156 residue (as a site of ubiquitin conjugation) were identified in $L$. donovani PTR1. This finding suggests that degradation of PTR1 during the stationary phase of growth is mediated by proteasomes, resulting in low levels of $\mathrm{H} 4$-biopterin, which promotes metacyclogenesis and subsequently results in highly infective parasite stages [60]. Two HIV-protease inhibitors, indinavir and saquinavir, have been shown to block proteasome functions; effects were observed on the growth of L. major and Leishmania infantum. After $24 \mathrm{~h}$ of treatment, both drugs exhibited dose-dependent antileishmanial activity, with lethal-dose values of $50 \%$ (LD50), $8.3 \mu \mathrm{M}$ and $7 \mu \mathrm{m}$ on $L$. major, and minor activity on $L$. infantum. These results suggest the potential use of these protease inhibitors against opportunistic infections in treated seropositive patients [61].

It has also been reported in $L$. donovani that the proteasome is involved in downregulation of methionine adenosyltransferase (MAT), an enzyme important for metabolic processes; its product, S-adenosylmethionine (AdoMet), plays a key role in trans-methylation, trans-sulfuration, and polyamine synthesis. The presence of proteasome inhibitors such as MG-132, MG-115, epoxomicin, and lactacystin in the culture medium prevented MAT degradation in both MAToverexpressing and "mock-transfected" leishmanial strains. The role of the ubiquitin (Ub) pathway in MAT downregulation was also supported by immunoprecipitation experiments. Immunoprecipitated MAT cross-reacted with antiUb antibodies, providing evidence of a proteasome-mediated downregulation of the leishmanial MAT abundance [62].

3.5. Trypanosomes. The Trypanosoma proteasome is the most intensely studied of the parasite proteasomes. The proteasome of Trypanosoma brucei was the first to be purified and characterized; however, its role in the biology of the parasite was not described [63]. Subsequent work showed that proteasome activity appears to be essential for cell-cycle progression, although participation seems to differ between the blood and procyclic forms. The amount of lactacystin needed to inhibit proliferation of procyclic forms concentrations was $5-10 \mu \mathrm{m}$, five times higher than was needed to inhibit the same process in blood forms. According to the authors, this difference in sensitivity to inhibitors could be explained by differences in the cell permeability. DNA analysis by flow cytometry showed that in the procyclic forms, lactacystin inhibits the progression of the cell cycle in the G2 and M phases, while in blood forms, makes it in G1/S, G2, and $\mathrm{M}$ phases. According to the same authors, in T. brucei, lactacystin at $1 \mu \mathrm{M}$ was unable to block the differentiation of blood forms to the procyclic stage [64]. These results suggest that in trypanosomes, proteasomes participate in the regulation of cyclin levels [65]. The 20S proteasome purified from procyclic and bloodstream forms has increased trypsinlike activity, unlike the eukaryotic proteasomes in which the chymotrypsin-like activity is higher. In addition, other differences between T. brucei and mammalian proteasomes have been found. (1) The $20 \mathrm{~S}$ proteasome of trypanosomes has a molecular weight of $630 \mathrm{kDa}$, while that of mammals is $700 \mathrm{kDa}$; (2) the 2D gels from the trypanosome $20 \mathrm{~S}$ proteasome have only 26 protein spots, fewer than observed in the $20 \mathrm{~S}$ proteasomes isolated from rat livers [66]; (3) although the morphology and size of the $T$. brucei proteasome are similar to those described in mammalian proteasomes, the pore diameter of the T. brucei $20 \mathrm{~S}$ proteasome is greater than that observed in the rat 20S proteasome; (4) polyclonal antibodies raised against the human $20 \mathrm{~S}$ proteasome crossreacted with the procyclic and bloodstreams forms of $T$. brucei 20 S proteasome; however, they strongly recognized rat $20 \mathrm{~S}$ proteasome. On the other hand, polyclonal antibodies obtained against the purified 20S proteasome isolated from blood forms of T. brucei $20 \mathrm{~S}$ also reacted with the purified $20 \mathrm{~S}$ proteasome isolated from procyclic forms of the parasite but not with the $20 \mathrm{~S}$ proteasome from rat erythrocytes. The $\alpha 5$ subunit of T. brucei proteasome has only $50 \%$ sequence identity with that of the rat proteasome [67].

A $20 \mathrm{~S}$ proteasome activator was also identified in procyclic and blood forms of T. brucei. In vitro, the $26 \mathrm{kDa}$ PA26 spontaneously polymerizes with proteasome $20 \mathrm{~S}$ to generate the activated $20 \mathrm{~S}$ proteasome [68]. Its human counterpart, PA28 $\alpha$, was as effective as PA26 in associating with and stimulating the enzymatic activity of the rat $20 \mathrm{~S}$ proteasome but was unable to activate the proteasome $20 \mathrm{~S}$ of T. brucei. Moreover, unlike mammalian and yeast proteasomes, the T. brucei proteasome is unable to degrade the mammalian ornithine decarboxylase-antizyme (ODC) complex, which catalyzes the first step in polyamine biosynthesis. This inability is a significant difference between trypanosomes and mammalian proteasomes [69]. Moreover, the functional characterization of 11 non-ATPase subunits (regulatory particles not-ATPase (Rpn)) in the 19S regulatory complex showed that when Rpn10 was deficient, a complex without Rpn was formed, but cell growth stopped. This structural dispensability but functional indispensability of Rpn10 constitutes another unique 
aspect of the T. brucei proteasome [70]. Similarly, proteomics and bioinformatic approaches have allowed the identification and mapping of T. brucei proteasomes [71].

Nine vinyl ester tripeptides selective for inhibition of mammalian proteasome trypsin-like activity have been tested for in vitro activity against T. brucei. Two showed trypanocidal activity in the low-micromolar range without displaying cytotoxicity against human cells; however, the compounds did not inhibit the trypsin-like activity of the trypanosome proteasome, although their effect correlates with inactivation of chymotrypsin-like activity. This finding suggests that the inhibitor sensitivities differ between mammalian and trypanosome proteasome. This difference may be exploited for rational antitrypanosomal drug development [72].

On the other hand, the role of the T. cruzi proteasomes in trypomastigote-to-amastigote differentiation has been clearly documented $[73,74]$. Lactacystin significantly blocked the transformation of trypomastigotes to amastigotes in axenic medium at $\mathrm{pH} 5.0$ [73]. The 20S proteasome was purified and characterized and shown to possess trypsin-like, chymotrypsin-like, and caspase-like activities. Treatment with lactacystin does not block cell invasion but strongly reduced discharge of the parasite. Similarly, leucine $\mathrm{C}^{14}$ metabolic labeling of trypomastigotes showed that proteolysis occurs during $T$. cruzi cell differentiation from trypomastigote to amastigote. This proteolytic pathway was blocked by proteasome inhibitors like lactacystin and vinyl sulphone, but not by serine or cysteinyl proteinase inhibitors, suggesting that the protein degradation that occurs during the parasite cell differentiation is proteasomedependent [74]. Then, during parasite cell differentiation at acidic $\mathrm{pH}$, an ATP-dependent proteolytic pathway was observed and $26 \mathrm{~S}$ proteasomes were identified and characterized by first time in a protozoan parasite [74]. Similarly, these authors demonstrated that cytoskeleton proteins, especially the paraflagellar rod antigen, were degraded by a proteasomedependent pathway. However, monoclonal antibodies raised against the $T$. cruzi $20 \mathrm{~S}$ proteasome have been observed by electron microscopy and confocal studies, the presence of proteasomes in the nucleus, cytoplasm, and kinetoplasts. These findings were confirmed by detection of proteasome chymotrypsin-like activities in the kinetoplast, isolated by Percoll gradients [75]. In mammalian cells, the UPS has been found in the outer mitochondrial membrane associated degradation (OMMAD) quality controls proteins localized to the OMM [76]. Then, at the outer membrane, the UPS may play a role in recycling either membrane-embedded or imported proteins [77]. The role that proteasomes fulfill in T. cruzi kinetoplast is still unknown. However, we could speculate that proteasomes may be involved not only in quality control of proteins but also in kinetoplast morphology changes that occur when trypomastigotes differentiate to amastigotes or epimastigotes differentiate to metacyclic trypomastigotes.

According to Cardoso et al. [78], inhibition of the ubiquitin-proteasome pathway in T. cruzi epimastigotes does not block adhesion but does disrupt cell division. In the same way, in vitro T. cruzi metacyclogenesis was strongly inhibited (95\%) by treatment with $5 \mu \mathrm{M}$ of lactacystin.
Proteasomal proteolysis during the in vitro metacyclogenesis of T. cruzi has also been studied. Cardoso et al. [79] demonstrated that proteasome-dependent proteolysis occurs during metacyclogenesis. No peaks of ubiquitin-mediated degradation were observed, and the profile of ubiquitinated conjugates was similar at all stages of differentiation; however, an analysis of carbonylated proteins showed significant variation in the levels of oxidized protein at the various stages of differentiation, and proteasome inhibition also increased oxidized-protein levels. These observations suggest that different proteasome complexes coexist during metacyclogenesis. The $20 \mathrm{~S}$ proteasome may be free or linked to regulatory particles (PA700, PA26, and PA200), at specific cell sites, and the coordinated action of these complexes would make it possible for proteolysis of ubiquitin-tagged proteins and oxidized proteins to cooccur in the cell. In addition, these findings strongly suggest that the coordinated series of biochemical adaptations occurring during T. cruzi metacyclogenesis may also be regulated by the activity of different proteasome complexes. These data also highlight the importance of ubiquitin-independent proteasomal degradation during metacyclogenesis [79]. The role of proteasomes in cell differentiation led us to propose this organelle as a trypanosome virulence factor [80].

Two genes encoding the $\alpha 1$ and $\alpha 6$ subunits of the $T$. cruzi proteasome have been cloned and characterized [81]. Considering that the most part structural studies have been performed in trypanosomes $[67,69,74,75]$, a subunits composition of human, yeast, and trypanosomes is shown in Table 1.

3.6. Plasmodium. The presence of the Plasmodium proteasome was first shown using inhibitors. Lactacystin inhibits the in vitro development of exoerythrocytic forms of Plasmodium berghei but does not inhibit sporozoite invasion of the host cell. The inhibitory effect of lactacystin is stagespecific, and although no infected rat survived, lactacystin reduced the parasitemia of the infected animals. The authors thus suggested the proteasome as a promising chemotherapeutic target [82]. On the other hand, lactacystin inhibited the growth of three different lines of Plasmodium falciparum at similar molar concentrations and was more effective against chloroquine-resistant parasites [83]. The genes encoding the $\beta$ subunits of $P$. falciparum 20 S proteasome have been already cloned [84].

Phosphoethanolamine methyltransferase (fepm), an enzyme of central importance in the serine decarboxylase phosphoethanolamine methyltransferase (SDPM) pathway, is negatively transcriptionally regulated and degraded by the proteasome in the presence of choline. Immunoblotting, pulse-chase, and chromatin immunoprecipitation experiments have shown that Pfpmt degradation occurred not only in wild-type cells but also in transgenic parasites that express Pfpmt constitutively. The proteasome inhibitor bortezomib blocked choline-mediated Pfpmt degradation. These data were the first evidence that a metabolite can mediate transcriptional regulation and proteasome degradation in Plasmodium [85]. 
TABLE 1: Proteasome subunits composition in diferent species of eukaryotic cells.

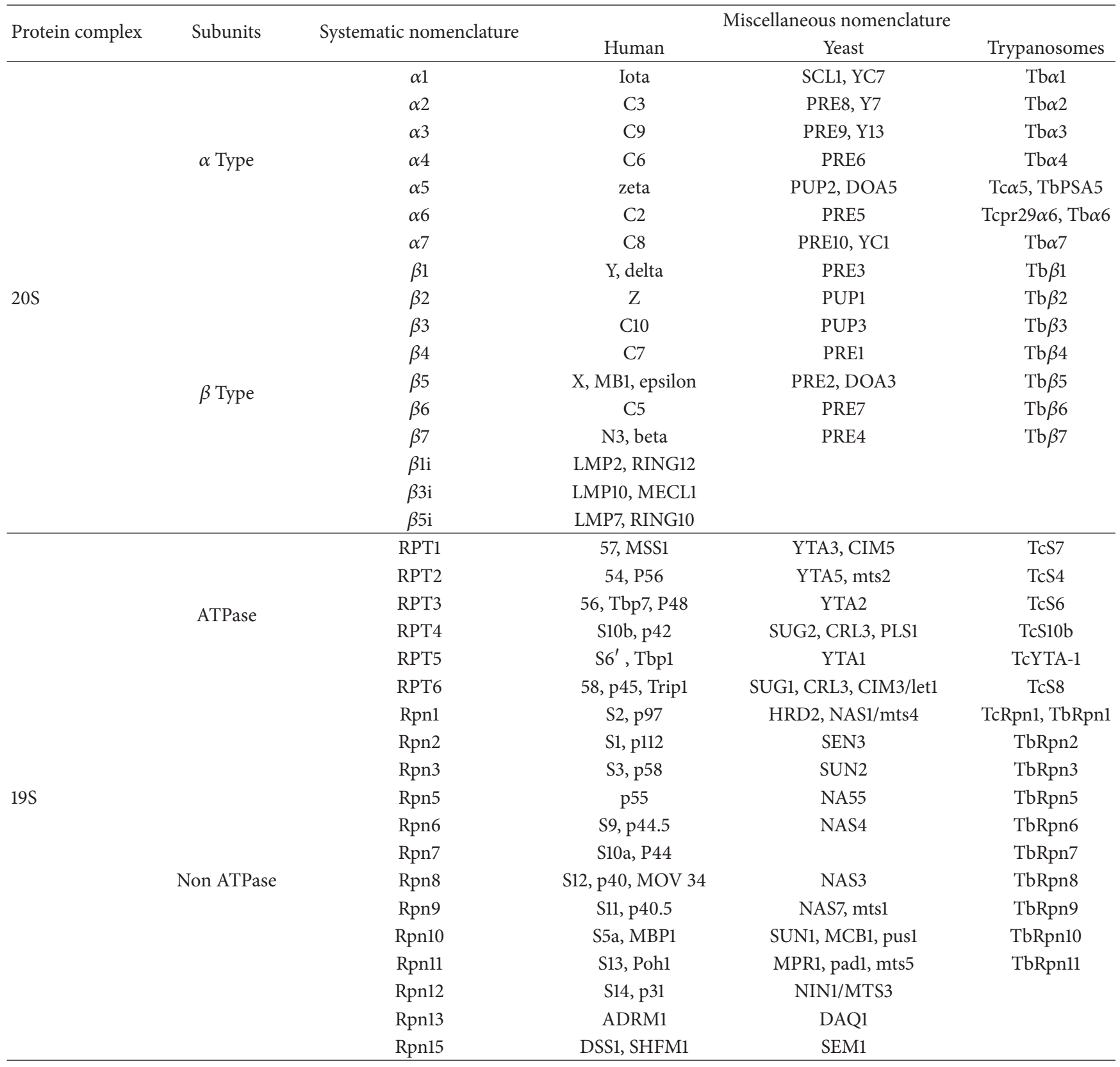

Gliotoxin (GTX), a metabolite of fungal origin, may have an in vitro antimalaria effect. GTX showed activity against chloroquine-sensitive and -resistant strains of $P$. falciparum. GTX cytotoxicity was significantly lower against normal liver cell lines [86]. According to the same researchers, GTX blocked chymotrypsin-like activity in the $P$. falciparum proteasome. In the same way, MLN-273, a proteasome inhibitor belonging to the peptidyl boronic acid family, has shown to inhibit the early intraerythrocytic stages of $P$. falciparum, as well as the exoerythrocytic stagesof $P$. berghei. The inhibitor did not affect the erythrocytes or the liver cells but caused a significant reduction in parasite protein degradation. According to these authors, the use of proteasome inhibitors as antineoplastic drugs suggests the possibility of malaria chemotherapy based on proteasome inhibitors [87]. From this perspective, proteasome inhibitors like bortezomib (Velcade: [(1R)-3-methyl-1-[[(2S)-1-oxo-3phenyl-2-[(pyrazinylcarbonyl) amino] propyl] amino] butyl] boronic acid), which has been approved for the treatment of patients with myeloma, and a similar boronate called Z-LeuLeu-Leu-B (OH) 2 (ZL3B), were assessed against four strains of $P$. falciparum (3D7, HB3, W2, and Dd2) which had different levels of sensitivity to antimalaria drugs like pyrimethamine and chloroquine. Both drugs were equally effective against susceptible and resistant parasites, blocking intraerythrocytic parasite development. These data strongly suggest that these 
drugs could be used alone or in association with malaria chemotherapy [88].

A comprehensive study of proteasome inhibitors active against $P$. falciparum laboratory strains and field isolates from Gabon showed that epoxomicin was highly active against $P$. falciparum and showed no signs of cross-resistance with similar drugs or any other proteasome inhibitor in an area with high-grade chloroquine resistance [89].

Although the Plasmodium proteasome has been suggested as potential antimalarial drug target, the toxicity of inhibitors has prevented validation of this enzyme in vivo. A screen of a library of 670 analogs of the recent US Food and Drug Administration-approved inhibitor, carfilzomib, was performed to identify compounds that selectively kill parasites. One of them, PR3, displayed significant parasitekilling activity in vitro but dramatically reduced toxicity in host cells. According to the authors, this parasite-specific toxicity was not due to selective targeting of the Plasmodium proteasome over the host proteasome but due to a lack of activity against one of the human-proteasome subunits. Subsequently, they used PR3 to significantly reduce parasite load in $P$. berghei infected mice without host toxicity, thus validating the proteasome as a viable antimalarial-drug target [90].

3.7. Toxoplasma. The Toxoplasma gondii proteasome has been examined in terms of its intracellular localization and enzymatic activity. Studies of immunofluorescence with antibodies against proteasome have shown that, unlike eukaryotic cells (in which the proteasome is located both in the nucleus and cytosol), in Toxoplasma, proteasomes are restricted to the cytosol. Chymotrypsin-like activity was detected, with $\mathrm{Km}$ values close to those observed in eukaryotic cells [91]. The pretreatment of free tachyzoites with proteasome inhibitors (10 $\mu \mathrm{m}$ lactacystin) or $5 \mu \mathrm{m}$ gliotoxin [92] did not block the entry of the parasite or the formation of the parasitophorous vacuole but did block intracellular parasite growth and DNA synthesis. However, Shaw et al. [93] showed that lactacystin $(2 \mu \mathrm{m})$ did not block parasite entry or the establishment of the parasitophorous vacuole but did inhibit parasite growth and daughter-cell budding, as well as DNA synthesis. Pretreatment of host cells with lactacystin did not block parasite entry or development. These results highlight the possible role of Toxoplasma proteasome activity in intracellular development and regulation of parasite replication. In the same way, parasite penetration of host cells was not modified by a high gliotoxin concentration $(1 \mu \mathrm{m})$, but replication was markedly decreased (approximately 50\% inhibition by $0.5 \mu \mathrm{m}$ gliotoxin). Gliotoxin reduced the chymotrypsin-like activity of the Toxoplasma proteasome with five times lower potency than in HeLa cells [92]. The major findings about the role of proteasomes in protozoan parasites are summarized in Figure 2.

\section{Concluding Remarks}

Protease activity is essential to many biological systems and processes. In parasites, proteases are essential for host-tissue degradation, immune evasion, and nutrition acquisition [94]. Until less than twenty years ago, the presence and biological role of proteasomes in parasites was not known.

Since the first report of proteasomes in protozoa [63], the first description of its biological function [73], and the first description of the existence of the $26 \mathrm{~S}$ proteasome in protozoa [74], several reports have confirmed the role of proteasomes in parasite biological processes such as differentiation, the cell cycle, proliferation, and encystation. Proliferation and differentiation are key steps in host colonization. Considering the importance of proteasomes in both processes in many different parasites such as Trypanosoma, Leishmania, Toxoplasma, and Entamoeba, parasite proteasomes might serve as virulence factors.

Despite the many parasitic biological phenomena in which the proteasome participates, information relating to how proteasome participates in such phenomena is not known. The majority of the parasite proteins that are degraded by proteasomes have not been identified. Proteasome targets and biological pathways involving proteasomes in key biological process remain to be clarified.

Proteasome inhibitors have been valuable research tools in cellular biology through the elucidation of important biological processes associated with the ubiquitin-proteasome protein-degradation pathway. The ubiquitin-proteasome system is a privileged pharmacological target for drug development due to the tremendous potential for intervention in multiple pathologies including cancer, neurodegenerative diseases, immune diseases, and infections. The pharmacological potential of the UPS was revealed after the unpredicted success of proteasome inhibitors for the treatment of some hematological malignancies.

Moreover, after US Food and Drug Administration approved bortezomib (Velcade) for the treatment of relapsed multiple myeloma, the proteasome has emerged as a new therapeutic target for diverse pathologies. Drug-discovery programs in academia and the pharmaceutical industry have developed a range of low-nanomolar natural and synthetic 20S-proteasome inhibitors and entered them in human clinical trials as significant anticancer and anti-inflammatory leads. The landscape of proteasome inhibitor-based therapeutics is quickly evolving, with promise beyond clinical oncology, and represents an exciting example of translational medicine.

Several pieces of evidence strongly suggest that the ubiquitin-proteasome pathway is also a viable parasitic therapeutic target. Research in recent years has shown that the proteasome is a valid drug target for sleeping sickness [72, 95, 96]. Although the structure of the trypanosome proteasome resembles that of its mammalian counterpart, the enzyme complexes differ from each other with respect to peptidase activity, substrate specificity, and inhibitor sensitivity. In addition, enzymatic analyses have demonstrated that the trypanosome and mammalian proteasomal functions are particularly sensitive to inhibition of the trypsin-like and chymotrypsin-like activities, respectively [97, 98]. Thus, compounds specifically targeting the trypsin-like activity of the trypanosome proteasome may constitute a basis for 


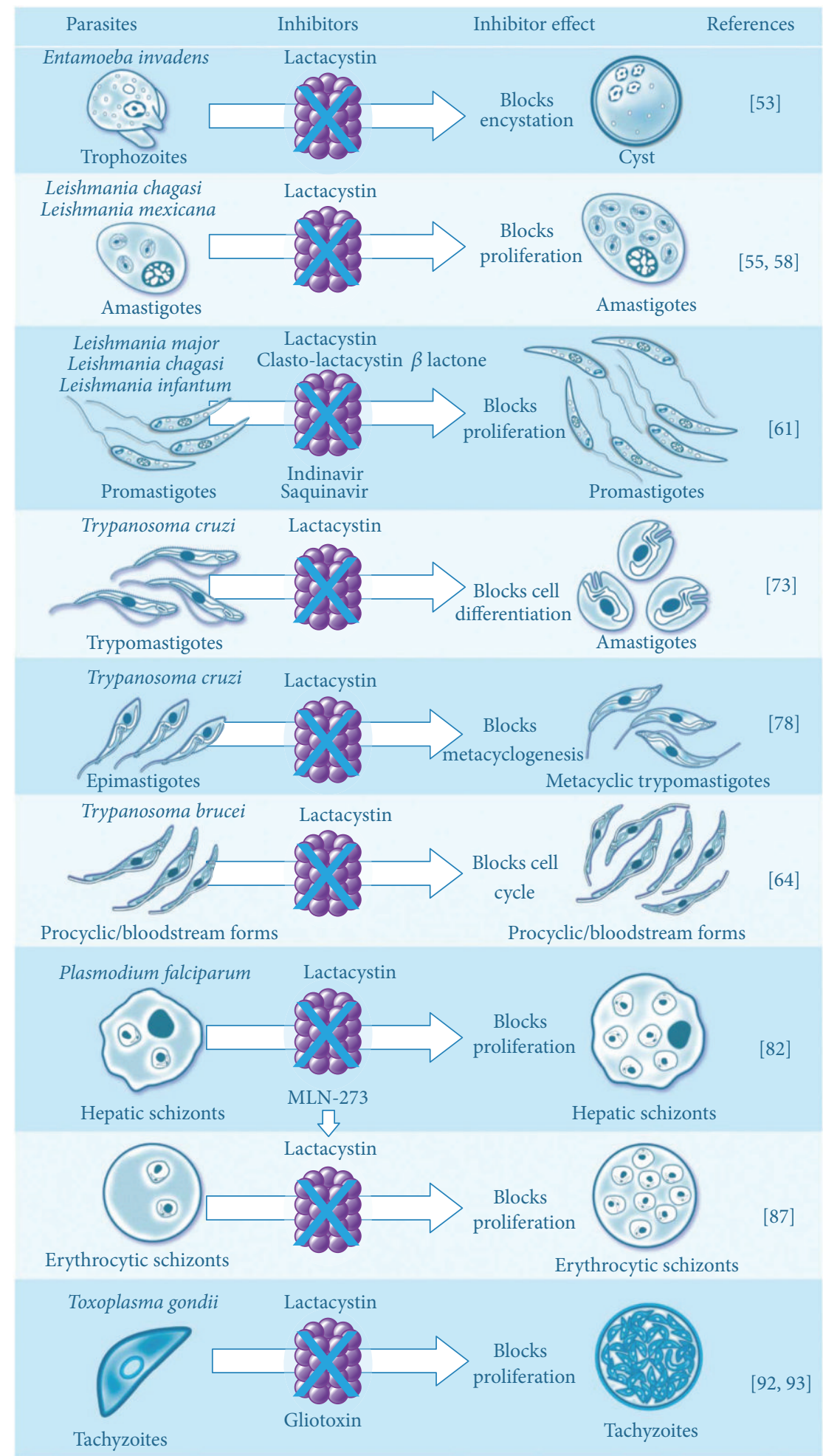

FIGURE 2: Role of the ubiquitin proteasome system in biology of protozoan parasites and effect of different proteasome inhibitors on proliferation and cell differentiation. 
rational antitrypanosomal drug development. However, the emergence and spread of $P$. falciparum resistance to existing antimalarials necessitates the search for novel drug targets and chemotherapeutic compounds. Inhibition of the proteasome is a promising strategy to develop novel antimalarial drugs.

Diseases caused by parasites affect hundreds of millions of people worldwide, with devastating health and economic effects; however, parasites have been largely neglected in terms of drug development because they affect poor people in poor regions of the world. Most of the drugs currently used to treat these diseases are decades old and have many limitations, including drug resistance. Proteasomes are a key organelle in parasite biology and virulence and appear to be an attractive new chemotherapeutic target.

\section{Conflict of Interests}

The authors declare that there is no conflict of interests regarding the publication of this paper.

\section{Acknowledgments}

The authors would like to thank Mr. You Garmendia for the support in designing and preparation of figures. This work was funded by the FONDECYT no. 1131007 to Jorge González.

\section{References}

[1] A. Ciehanover, Y. Hod, and A. Hershko, "A heat-stable polypeptide component of an ATP-dependent proteolytic system from reticulocytes," Biochemical and Biophysical Research Communications, vol. 81, no. 4, pp. 1100-1105, 1978.

[2] A. Ciechanover, H. Heller, S. Elias, A. L. Haas, and A. Hershko, "ATP-dependent conjugation of reticulocyte proteins with the polypeptide required for protein degradation," Proceedings of the National Academy of Sciences of the United States of America, vol. 77, no. 3, pp. 1365-1368, 1980.

[3] A. Hershko, A. Ciechanover, H. Heller, A. L. Haas, and I. A. Rose, "Proposed role of ATP in protein breakdown: conjugation of protein with multiple chains of the polypeptide of ATPdependent proteolysis," Proceedings of the National Academy of Sciences of the United States of America, vol. 77, no. 4, pp. 17831786, 1980.

[4] A. Hershko, "Lessons from the discovery of the ubiquitin system," Trends in Biochemical Sciences, vol. 21, no. 11, pp. 445449, 1996.

[5] R. Hough, G. Pratt, and M. Rechsteiner, "Ubiquitin-lysozyme conjugates. Identification and characterization of an ATPdependent protease from rabbit reticulocyte lysates," Journal of Biological Chemistry, vol. 261, no. 5, pp. 2400-2408, 1986.

[6] A. Ciechanover, "The ubiquitin proteolytic system: from a vague idea, through basic mechanisms, and onto human diseases and drug targeting," Neurology, vol. 66, no. 2, pp. S7-S19, 2006.

[7] Y. Saeki and K. Tanaka, "Assembly and function of the proteasome," in Ubiquitin Family Modifiers and the Proteasome, vol. 832 of Methods in Molecular Biology, pp. 315-337, Humana Press, New Jersey, NJ, USA, 2012.
[8] R. E. Valas and P. E. Bourne, "Rethinking proteasome evolution: two novel bacterial proteasomes," Journal of Molecular Evolution, vol. 66, no. 5, pp. 494-504, 2008.

[9] K. Tanaka, “The proteasome: from basic mechanisms to emerging roles," Keio Journal of Medicine, vol. 62, no. 1, pp. 1-12, 2013.

[10] R. Huber, J. Löwe, D. Stock, B. Jap, P. Zwickl, and W. Baumeister, "Crystal structure of the 20S proteasome from the archaeon $T$. acidophilum at 3.4 resolution," Science, vol. 268, pp. 533-539, 1995.

[11] M. Groll, L. Ditzel, J. Löwe et al., "Structure of 20S proteasome from yeast at $2.4 \AA$ resolution," Nature, vol. 386, no. 6624, pp. 463-471, 1997.

[12] M. Groll, M. Bajorek, A. Köhler et al., "A gated channel into the proteasome core particle," Nature Structural Biology, vol. 7, no. 11, pp. 1062-1067, 2000.

[13] M. Rechsteiner and C. P. Hill, "Mobilizing the proteolytic machine: cell biological roles of proteasome activators and inhibitors," Trends in Cell Biology, vol. 15, no. 1, pp. 27-33, 2005.

[14] D. Voges, P. Zwickl, and W. Baumeister, "The $26 \mathrm{~S}$ proteasome: a molecular machine designed for controlled proteolysis," Annual Review of Biochemistry, vol. 68, pp. 1015-1068, 1999.

[15] C. P. Hill, E. I. Masters, and F. G. Whitby, "The 11S regulators of 20 S proteasome activity," Current Topics in Microbiology and Immunology, vol. 268, pp. 73-89, 2002.

[16] V. Ustrell, L. Hoffman, G. Pratt, and M. Rechsteiner, "Pa200, a nuclear proteasome activator involved in DNA repair," The EMBO Journal, vol. 21, no. 13, pp. 3516-3525, 2002.

[17] J. Blickwedehl, M. Agarwal, C. Seong et al., "Role for proteasome activator PA200 and postglutamyl proteasome activity in genomic stability," Proceedings of the National Academy of Sciences of the United States of America, vol. 105, no. 42, pp. 16165-16170, 2008.

[18] A. F. Savulescu and M. H. Glickman, "Proteasome activator 200: the heat is on," Molecular and Cellular Proteomics, vol. 10, no. 5, 2011.

[19] M. Fehlker, P. Wendler, A. Lehmann, and C. Enenkel, "Blm3 is part of nascent proteasomes and is involved in a late stage of nuclear proteasome assembly," The EMBO Reports, vol. 4, no. 10, pp. 959-963, 2003.

[20] C. Concannon and R. S. Lahue, "Nucleotide excision repair and the $26 \mathrm{~S}$ proteasome function together to promote trinucleotide repeat expansions," DNA Repair, vol. 13, no. 1, pp. 42-49, 2014.

[21] A. Lehmann, K. Jechow, and C. Enenkel, "Blm10 binds to preactivated proteasome core particles with open gate conformation," The EMBO Reports, vol. 9, no. 12, pp. 1237-1243, 2008.

[22] M.-X. Qian, Y. Pang, C. H. Liu et al., "XAcetylation-mediated proteasomal degradation of core histones during DNA repair and spermatogenesis," Cell, vol. 153, no. 5, pp. 1012-1024, 2013.

[23] K. Sadre-Bazzaz, F. G. Whitby, H. Robinson, T. Formosa, and C. P. Hill, "Structure of a Blm10 complex reveals common mechanisms for proteasome binding and gate opening," Molecular Cell, vol. 37, no. 5, pp. 728-735, 2010.

[24] B. M. Stadtmueller and C. P. Hill, "Proteasome activators," Molecular Cell, vol. 41, no. 1, pp. 8-19, 2011.

[25] A. Ciechanover and A. Stanhill, "The complexity of recognition of ubiquitinated substrates by the $26 \mathrm{~S}$ proteasome," Biochimica et Biophysica Acta, vol. 1843, no. 1, pp. 86-96, 2014.

[26] D. M. Smith, S.-C. Chang, S. Park, D. Finley, Y. Cheng, and A. L. Goldberg, "Docking of the proteasomal ATPases' carboxyl termini in the $20 \mathrm{~S}$ proteasome's $\alpha$ ring opens the gate for substrate entry," Molecular Cell, vol. 27, no. 5, pp. 731-744, 2007. 
[27] D. Bech-Otschir, A. Helfrich, C. Enenkel et al., "Polyubiquitin substrates allosterically activate their own degradation by the 26 S proteasome," Nature Structural \& Molecular Biology, vol. 16, no. 2, pp. 219-225, 2009.

[28] P. Śledź, F. Förster, and W. Baumeister, "Allosteric effects in the regulation of $26 \mathrm{~S}$ proteasome activities," Journal of Molecular Biology, vol. 425, no. 9, pp. 1415-1423, 2013.

[29] A. M. Ruschak and L. E. Kay, "Proteasome allostery as a population shift between interchanging conformers," Proceedings of the National Academy of Sciences of the United States of America, vol. 109, no. 50, pp. E3454-E3462, 2012.

[30] A. M. Weissman, "Themes and variations on ubiquitylation," Nature Reviews Molecular Cell Biology, vol. 2, no. 3, pp. 169-178, 2001.

[31] L. A. Passmore and D. Barford, "Getting into position: the catalytic mechanisms of protein of protein ubiquitylation," Biochemical Journal, vol. 379, no. 3, pp. 513-525, 2004.

[32] P.-M. Kloetzel, "Antigen processing by the proteasome," Nature Reviews Molecular Cell Biology, vol. 2, no. 3, pp. 179-187, 2001.

[33] J. M. Baugh, E. G. Viktorova, and E. V. Pilipenko, "Proteasomes can degrade a significant proportion of cellular proteins independent of ubiquitination," Journal of Molecular Biology, vol. 386, no. 3, pp. 814-827, 2009.

[34] S. S. Wing, "Deubiquitinating enzymes-The importance of driving in reverse along the ubiquitin-proteasome pathway," International Journal of Biochemistry and Cell Biology, vol. 35, no. 5, pp. 590-605, 2003.

[35] M. Pagano, "Cell cycle regulation by the ubiquitin pathway," The FASEB Journal, vol. 11, no. 13, pp. 1067-1075, 1997.

[36] H. L. Pahl and P. A. Baeuerle, "Control of gene expression by proteolysis," Current Opinion in Cell Biology, vol. 8, no. 3, pp. 340-347, 1996.

[37] M. Piccinini, M. Mostert, and M. T. Rinaudo, "Proteasomes as drug targets," Current Drug Targets, vol. 4, no. 8, pp. 657-671, 2003.

[38] I. M. Konstantinova, A. S. Tsimokha, and A. G. Mittenberg, "Role of proteasomes in cellular regulation," International Review of Cell and Molecular Biology, vol. 267, pp. 59-124, 2008.

[39] W. Ward, L. Alvarado, N. D. Rawlings, J. C. Engel, C. Franklin, and J. H. McKerrow, "A primitive enzyme for a primitive cell: the protease required for excystation of Giardia," Cell, vol. 89, no. 3, pp. 437-444, 1997.

[40] J. R. Forney, S. Yang, and M. C. Healey, "Protease activity associated with excystation of Cryptosporidium parvum oocysts," Journal of Parasitology, vol. 82, no. 6, pp. 889-892, 1996.

[41] A. Makioka, M. Kumagai, S. Kobayashi, and T. Takeuchi, "Entamoeba invadens: cysteine protease inhibitors block excystation and metacystic development," Experimental Parasitology, vol. 109, no. 1, pp. 27-32, 2005.

[42] A. Paugam, A.-L. Bulteau, J. Dupouy-Camet, C. Creuzet, and B. Friguet, "Characterization and role of protozoan parasite proteasomes," Trends in Parasitology, vol. 19, no. 2, pp. 55-59, 2003.

[43] B. Dahlmann, "Role of proteasomes in disease," BMC Biochemistry, vol. 8, no. 1, article S3, 2007.

[44] P. A. Chung, J. Johnson, N. V. Khramtsov, and S. J. Upton, "Cloning and molecular characterization of a gene encoding a cryptosporidium parvum putative 20 s proteasome $\beta 1$-type subunit," Mitochondrial DNA, vol. 11, no. 3-4, pp. 309-314, 2000.
[45] V. Emmerlich, U. Santarius, T. Bakker-Grunwald, and H. Scholze, "Isolation and subunit composition of the $20 \mathrm{~S}$ proteasome of Giardia lamblia," Molecular and Biochemical Parasitology, vol. 100, no. 1, pp. 131-134, 1999.

[46] J. L. Bouzatrid, L. K. McNeilrid, H. M. Robertson et al., "Phylogenomic analysis of the $\alpha$ proteasome gene family from early-diverging eukaryotes," Journal of Molecular Evolution, vol. 51, no. 6, pp. 532-543, 2000.

[47] V.Emmerlich, H. Scholze, F. D. Gillin, and T. Bakker-Grunwald, "Characterization of a proteasome $\alpha$-chain from Giardia lamblia," Parasitology Research, vol. 87, no. 2, pp. 112-115, 2001.

[48] S. Stefanic, D. Palm, S. G. Svärd, and A. B. Hehl, "Organelle proteomics reveals cargo maturation mechanisms associated with Golgi-like encystation vesicles in the early-diverged protozoan Giardia lamblia," Journal of Biological Chemistry, vol. 281, no. 11, pp. 7595-7604, 2006.

[49] H. Scholze, S. Frey, Z. Cejka, and T. Bakker-Grunwald, "Evidence for the existence of both proteasomes and a novel high molecular weight peptidase in Entamoeba histolytica," The Journal of Biological Chemistry, vol. 271, no. 11, pp. 6212-6216, 1996.

[50] J. Tolstrup, E. Krause, E. Tannich, and I. Bruchhaus, "Proteomic analysis of Entamoeba histolytica," Parasitology, vol. 134, no. 2, pp. 289-298, 2007.

[51] R. Sánchez, A. Alagón, and R. P. Stock, "Entamoeba histolytica: intracellular distribution of the proteasome," Experimental Parasitology, vol. 102, no. 3-4, pp. 187-190, 2002.

[52] D. E. Bosch and D. P. Siderovski, "Structural determinants of ubiquitin conjugation in Entamoeba histolytica," Journal of Biological Chemistry, vol. 288, no. 4, pp. 2290-2302, 2013.

[53] J. González, G. Bai, U. Frevert, E. J. Corey, and D. Eichinger, "Proteasome-dependent cyst formation and stage-specific ubiquitin mRNA accumulation in Entamoeba invadens," European Journal of Biochemistry, vol. 264, no. 3, pp. 897-904, 1999.

[54] A. Makioka, M. Kumagai, H. Ohtomo, S. Kobayashi, and T. Takeuchi, "Effect of proteasome inhibitors on the growth, encystation, and excystation of Entamoeba histolytica and Entamoeba invadens," Parasitology Research, vol. 88, no. 5, pp. 454459, 2002.

[55] C. D. Robertson, "The Leishmania mexicana proteasome," Molecular and Biochemical Parasitology, vol. 103, no. 1, pp. 4960, 1999.

[56] C. B. V. Christensen, L. Jørgensen, A. T. R. Jensen et al., "Molecular characterization of a Leishmania donovanii cDNA clone with similarity to human 20 S proteasome a-type subunit," Biochimica et Biophysica Acta-Molecular Basis of Disease, vol. 1500, no. 1, pp. 77-87, 2000.

[57] B. Couvreur, A. Bollen, J.-C. Dujardin, and D. Le Ray, "More panantigens in Leishmania," Trends in Parasitology, vol. 17, no. 2, pp. 63-64, 2001.

[58] I. Silva-Jardim, M. Fátima Horta, and F. J. Ramalho-Pinto, “The Leishmania chagasi proteasome: role in promastigotes growth and amastigotes survival within murine macrophages," Acta Tropica, vol. 91, no. 2, pp. 121-130, 2004.

[59] P. Dubessay, C. Blaineau, P. Bastien et al., "Cell cycle-dependent expression regulation by the proteasome pathway and characterization of the nuclear targeting signal of a Leishmania major Kin-13 kinesin," Molecular Microbiology, vol. 59, no. 4, pp. 11621174, 2006. 
[60] P. Kumar, S. Sundar, and N. Singh, "Degradation of pteridine reductase 1 (PTR1) enzyme during growth phase in the protozoan parasite Leishmania donovani," Experimental Parasitology, vol. 116, no. 2, pp. 182-189, 2007.

[61] D. Savoia, T. Allice, and P. A. Tovo, "Antileishmanial activity of HIV protease inhibitors," International Journal of Antimicrobial Agents, vol. 26, no. 1, pp. 92-94, 2005.

[62] Y. Pérez-Pertejo, R. Álvarez-Velilla, C. G. Estrada, R. BalañaFouce, and R. M. Reguera, "Leishmania donovani: proteasomemediated down-regulation of methionine adenosyltransferase," Parasitology, vol. 138, no. 9, pp. 1082-1092, 2011.

[63] H. Shao-Bing, W.-Y. To, T. T. Nguyen, M.-L. Wong, and C. C. Wang, "Purification and characterization of proteasomes from Trypanosoma brucei," Molecular and Biochemical Parasitology, vol. 78, no. 1-2, pp. 33-46, 1996.

[64] M. C. Mutomba and C. C. Wang, "The role of proteolysis during differentiation of Trypanosoma brucei from the bloodstream to the procyclic form," Molecular and Biochemical Parasitology, vol. 93, no. 1, pp. 11-22, 1998.

[65] J. J. van Hellemond and J. C. Mottram, "The CYC3 gene of Trypanosoma brucei encodes a cyclin with a short half-life," Molecular and Biochemical Parasitology, vol. 111, no. 2, pp. 275282, 2000.

[66] L. Huang, M. Shen, I. Chernushevich, A. L. Burlingame, C. C. Wang, and C. D. Robertson, "Identification and isolation of three proteasome subunits and their encoding genes from Trypanosoma brucei," Molecular and Biochemical Parasitology, vol. 102, no. 2, pp. 211-223, 1999.

[67] Y. Yao, C. R. Toth, L. Huang et al., “ $\alpha 5$ subunit in Trypanosoma brucei proteasome can self-assemble to form a cylinder of four stacked heptamer rings," Biochemical Journal, vol. 344, no. 2, pp. 349-358, 1999.

[68] Y. Yao, L. Huang, A. Krutchinsky et al., "Structural and functional characterizations of the proteasome-activating protein PA26 from Trypanosoma brucei," Journal of Biological Chemistry, vol. 274, no. 48, pp. 33921-33930, 1999.

[69] Z. Li and C. C. Wang, "Functional characterization of the 11 non-ATPase subunit proteins in the trypanosome $19 \mathrm{~S}$ proteasomal regulatory complex," Journal of Biological Chemistry, vol. 277, no. 45, pp. 42686-42693, 2002.

[70] Z. Li, C.-B. Zou, Y. Yao et al., "An easily dissociated $26 \mathrm{~S}$ proteasome catalyzes an essential ubiquitin-mediated protein degradation pathway in Trypanosoma brucei," Journal of Biological Chemistry, vol. 277, no. 18, pp. 15486-15498, 2002.

[71] L. Huang, R. J. Jacob, S. C.-H. Pegg et al., "Functional assignement of the 20S proteasome from Trypanosoma brucei using mass spectrometry and new bioinformatics approaches," The Journal of Biological Chemistry, vol. 276, no. 30, pp. 2832728339, 2001.

[72] D. Steverding, A. Baldisserotto, X. Wang, and M. Marastoni, "Trypanocidal activity of peptidyl vinyl ester derivatives selective for inhibition of mammalian proteasome trypsin-like activity," Experimental Parasitology, vol. 128, no. 4, pp. 444-447, 2011.

[73] J. González, F. J. Ramalho-Pinto, U. Frevert et al., "Proteasome activity is required for the stage-specific transformation of a protozoan parasite," The Journal of Experimental Medicine, vol. 184, no. 5, pp. 1909-1918, 1996.

[74] J. de Diego, J. M. Katz, P. Marshall et al., "The ubiquitinproteasome pathway plays an essential role in proteolysis during Trypanosoma cruzi remodeling," Biochemistry, vol. 40, no. 4, pp. 1053-1062, 2001.
[75] B. Gutiérrez, L. Osorio, M. C. M. Motta et al., "Molecular characterization and intracellular distribution of the alpha 5 subunit of Trypanosoma cruzi 20S proteasome," Parasitology International, vol. 58, no. 4, pp. 367-374, 2009.

[76] A. Neutzner, R. J. Youle, and M. Karbowski, "Outer mitochondrial membrane protein degradation by the proteasome," Novartis Foundation Symposium, vol. 287, pp. 4-14, 2007.

[77] N. Livnat-Levanon and M. H. Glickman, "Ubiquitinproteasome system and mitochondria-reciprocity," Biochimica et Biophysica Acta-Gene Regulatory Mechanisms, vol. 1809, no. 2, pp. 80-87, 2011.

[78] J. Cardoso, M. J. Soares, R. F. S. Menna-Barreto et al., "Inhibition of proteasome activity blocks Trypanosoma cruzi growth and metacyclogenesis," Parasitology Research, vol.103, no. 4, pp. 941951, 2008.

[79] J. Cardoso, C. D. P. Lima, T. Leal et al., "Analysis of proteasomal proteolysis during the in vitro metacyclogenesis of Trypanosoma cruzi," PLoS ONE, vol. 6, no. 6, Article ID e21027, 2011.

[80] L. Osorio, I. Ríos, B. Gutiérrez, and J. González, "Virulence factors of Trypanosoma cruzi: who is who?" Microbes and Infection, vol. 14, no. 15, pp. 1390-1402, 2012.

[81] D. C. Bartholomeu, J. A. N. Batista, M. H. Vainstein, B. D. Lima, and M. C. de Sá, "Molecular cloning and characterization of a gene encoding the $29-\mathrm{kDa}$ proteasome subunit from Trypanosoma cruzi," Molecular Genetics and Genomics, vol. 265, no. 6, pp. 986-992, 2001.

[82] S. M. Gantt, J. M. Myung, M. R. S. Briones et al., "Proteasome inhibitors block development of Plasmodium spp," Antimicrobial Agents and Chemotherapy, vol. 42, no. 10, pp. 2731-2738, 1998.

[83] G. Certad, A. Abrahem, and E. Georges, "Cloning and partial characterization of the proteasome S4 ATPase from Plasmodium falciparum," Experimental Parasitology, vol. 93, no. 3, pp. 123-131, 1999.

[84] G.-D. Li, J.-L. Li, M. Mugthin, and S. A. Ward, "Molecular cloning of a gene encoding a $20 \mathrm{~S}$ proteasome $\beta$ subunit from Plasmodium falciparum," International Journal for Parasitology, vol. 30, no. 6, pp. 729-733, 2000.

[85] W. H. Witola and C. B. Mamoun, "Choline induces transcriptional repression and proteasomal degradation of the malarial phosphoethanolamine methyltransferase," Eukaryotic Cell, vol. 6, no. 9, pp. 1618-1624, 2007.

[86] T. Hatabu, M. Hagiwara, N. Taguchi et al., "Plasmodium falciparum: the fungal metabolite gliotoxin inhibits proteasome proteolytic activity and exerts a plasmodicidal effect on $P$. falciparum," Experimental Parasitology, vol. 112, no. 3, pp. 179$183,2006$.

[87] C. Lindenthal, N. Weich, Y. S. Chia, V. Heussler, and M. Q. Klinkert, "The proteasome inhibitor MLN-273 blocks exoerythrocytic and erythrocytic development of Plasmodium parasites," Parasitology, vol. 131, no. 1, pp. 37-44, 2005.

[88] J. M. Reynolds, K. El Bissati, J. Brandenburg, A. Günzl, and C. B. Mamoun, "Antimalarial activity of the anticancer and proteasome inhibitor bortezomib and its analog ZL3B," BMC Clinical Pharmacology, vol. 7, article 13, 2007.

[89] A. Kreidenweiss, P. G. Kremsner, and B. Mordmüller, "Comprehensive study of proteasome inhibitors against Plasmodium falciparum laboratory strains and field isolates from Gabon," Malaria Journal, vol. 7, article 187, 2008.

[90] H. Li, E. L. Ponder, M. Verdoes et al., "Validation of the proteasome as a therapeutic target in Plasmodium using an 
epoxyketone inhibitor with parasite-specific toxicity," Chemistry \& Biology, vol. 19, no. 12, pp. 1535-1545, 2012.

[91] A. Paugam, C. Creuzet, J. Dupouy-Camet, and M. P. Roisin, "Evidence for the existence of a proteasome in Toxoplasma gondii: intracellular localization and specific peptidase activities," Parasite, vol. 8, no. 4, pp. 267-273, 2001.

[92] A. Paugam, C. Creuzet, J. Dupouy-Camet, and M. Roisin, "In vitro effects of gliotoxin, a natural proteasome inhibitor, on the infectivity and proteolytic activity of Toxoplasma gondii," Parasitology Research, vol. 88, no. 8, pp. 785-787, 2002.

[93] M. K. Shaw, C. Y. He, D. S. Roos, and L. G. Tilney, "Proteasome inhibitors block intracellular growth and replication of Toxoplasma gondii," Parasitology, vol. 121, no. 1, pp. 35-47, 2000.

[94] J. H. McKerrow, C. Caffrey, B. Kelly, P. Loke, and M. Sajid, "Proteases in parasitic diseases," Annual Review of Pathology, vol. 1, pp. 497-536, 2006.

[95] D. Steverding, X. Wang, B. C. Potts, and M. A. Palladino, "Trypanocidal activity of $\beta$-lactone- $\gamma$-lactam proteasome inhibitors," Planta Medica, vol. 78, no. 2, pp. 131-134, 2012.

[96] C. C. Wang, Z. Bozdech, C.-L. Liu et al., "Biochemical analysis of the 20 S proteasome of Trypanosoma brucei," Journal of Biological Chemistry, vol. 278, no. 18, pp. 15800-15808, 2003.

[97] R. J. Glenn, A. J. Pemberton, H. J. Royle et al., “Trypanocidal effect of $\alpha^{\prime}, \beta^{\prime}$-epoxyketones indicates that trypanosomes are particularly sensitive to inhibitors of proteasome trypsin-like activity," International Journal of Antimicrobial Agents, vol. 24, no. 3, pp. 286-289, 2004.

[98] D. Steverding, R. W. Spackman, H. J. Royle, and R. J. Glenn, "Trypanocidal activities of trileucine methyl vinyl sulfone proteasome inhibitors," Parasitology Research, vol. 95, no. 1, pp. 73-76, 2005. 

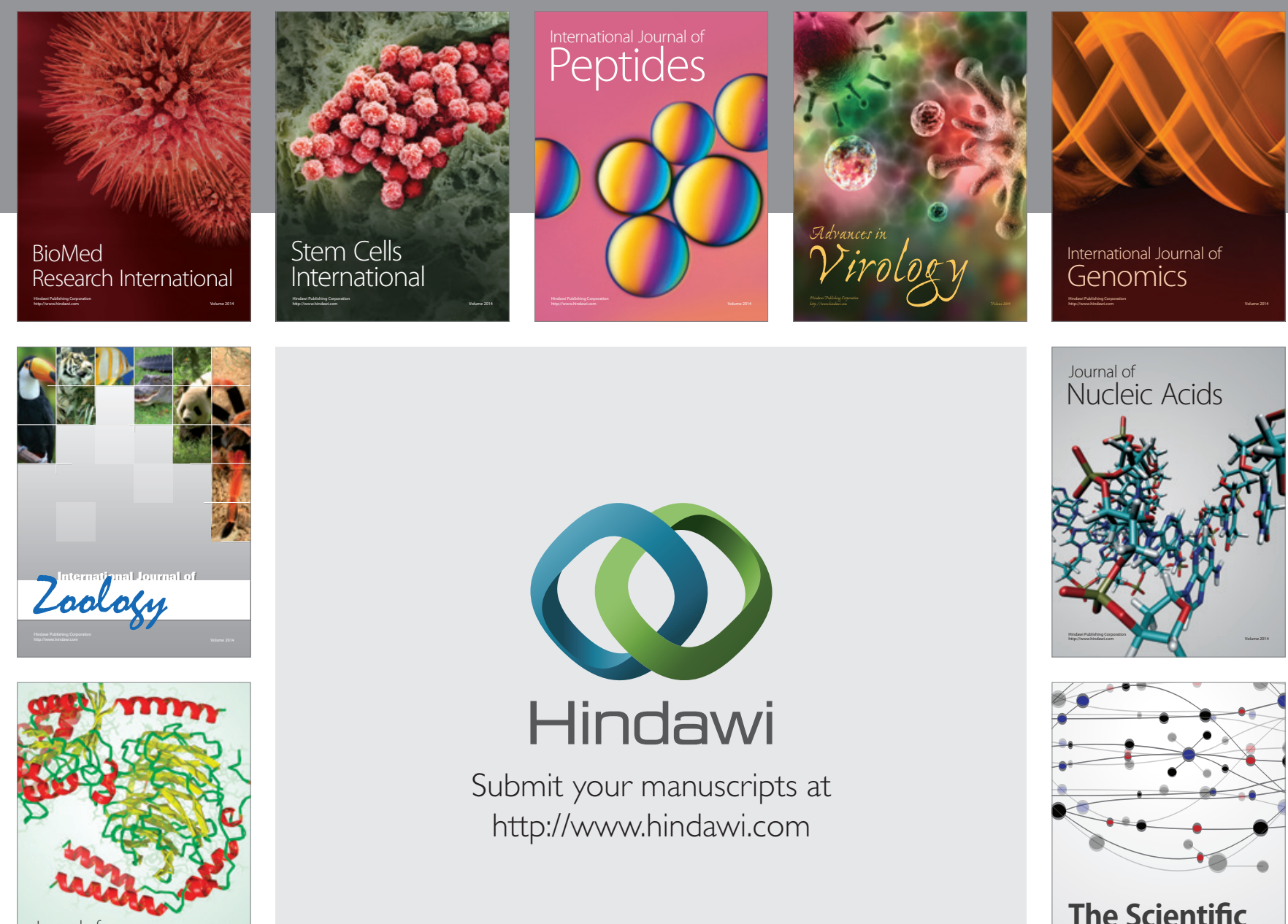

Submit your manuscripts at

http://www.hindawi.com

Journal of
Signal Transduction
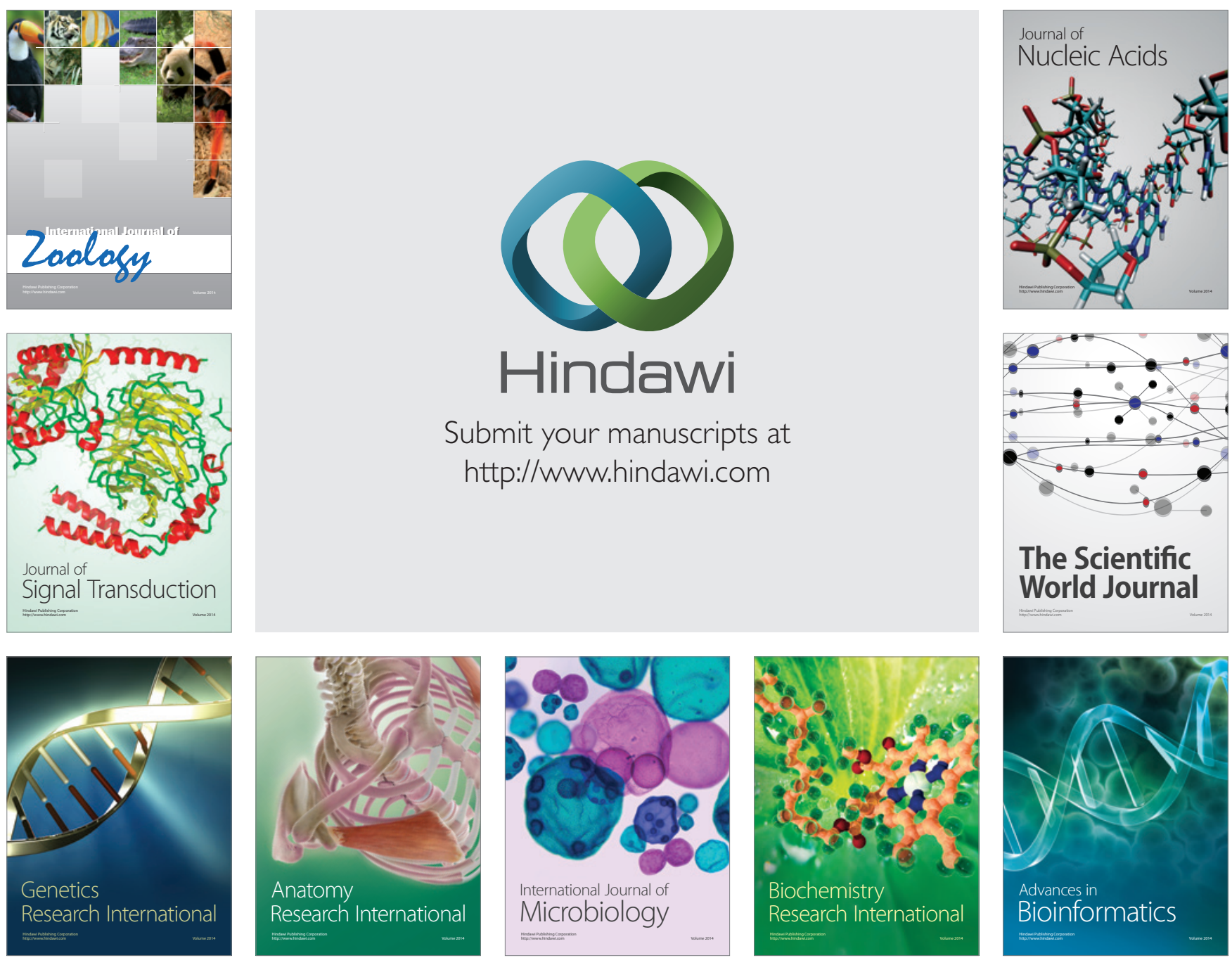

The Scientific World Journal
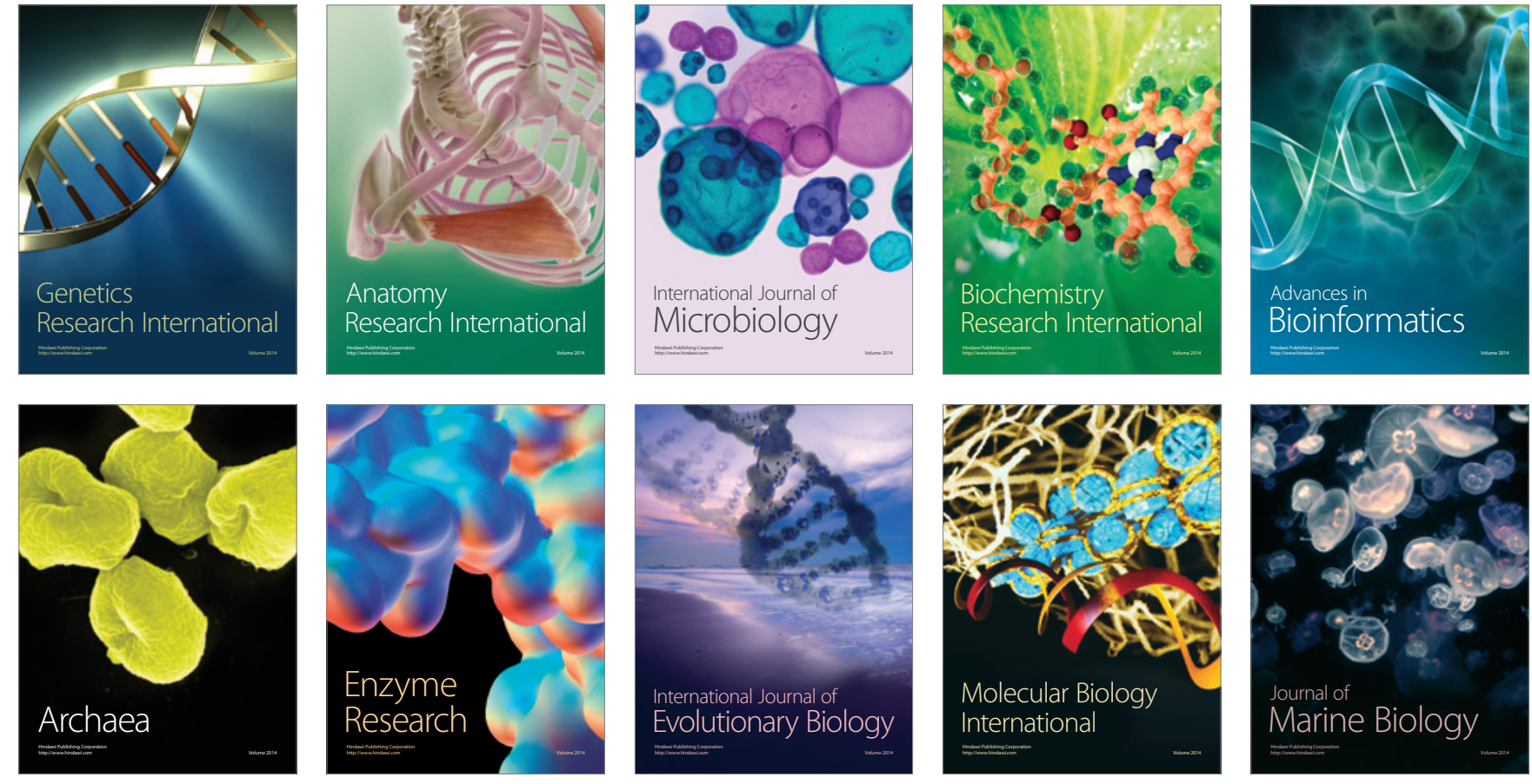